sented by Sir John Pendry (Imperial College London) for the Anniversary Prize, whose talk is titled "Metamaterials open new horizons in electromagnetism," and by Manuel Bibes (CNRS/ Thales, France), who has received the EU-40 Materials Prize. Bibes will address "Nanoferronics: controlling charge and spin currents by ferroic orders."
The E-MRS Fall Meeting will consist of 14 thematic symposia, a plenary session, and satellite events. It is chaired by George Kiriakidis of the Foundation for Research and TechnologyHellas (FORTH), Greece; Giuseppina Padeletti of ISMN-CNR, Italy; Witold Lojkowski of the Polish Academy of Sciences and Bialystok University of
Technology, Poland; and Wolfgang Jaeger of Christian-Albrechts-Universität, Germany.

The abstract deadline is June 10, 2013.

More information on both Meetings can be accessed at www.emrsstrasbourg.com.

\title{
ICMAT2013 to be held in Singapore
}

www.mrs.org.sg/icmat2013

T

he 7th International Conference on

Materials for Advanced Technologies (ICMAT2013) will be held on June 30 to July 5, 2013 in Singapore, hosted by the Materials Research Society of Singapore. The conference is chaired by B.V.R. Chowdari with Technical Chair Yuan Ping Feng, both from the National University of Singapore.

Symposia will cover Energy/Water Aspects, Nanoscience and Technology, Functional Materials, and Bio/Soft Materials.

Nobel laureate Alan Heeger (University of California-Santa Barbara) will present a plenary lecture on "The role of the Heisenberg unceertainty principle in bulk heterojunction solar cells" as well as a public lecture on "Creativity, discovery and risk: Nobel prizes past and future."

Nobel laureate Yuan-Tseh Lee (Academia Sinica, Taiwan) will also present a plenary and public lecture. The first lecture is titled "Elementary processes involved in matrix-assisted laser desorption ionization," and the public lecture will be on "Sustainable development of human society."

Other plenary speakers include Gabriel Aeppli (University College London, United Kingdom) addressing "The next life of silicon"; Andy Hor (National University of Singapore and Institute of Materials and Research Engineering
A*Star, Singapore), "Molecular design of catalytic materials through intelligent hybrid ligands"; and Helmuth Mohwald (Max-Planck-InstiMRS tute of Colloids and Interfaces, Germany), "Films and capsules from polyelectrolytes and nanoparticles for biomedical and materials applications."

More information can be accessed from the conference website www.mrs. org.sg/icmat 2013 or by contacting Secretariat Office, Meeting Matters International, 1 Commonwealth Lane, \#06-23 One Commonwealth, Singapore 149544; tel. 65 6472 3108; or email icmat2013@ meetmatt.net.

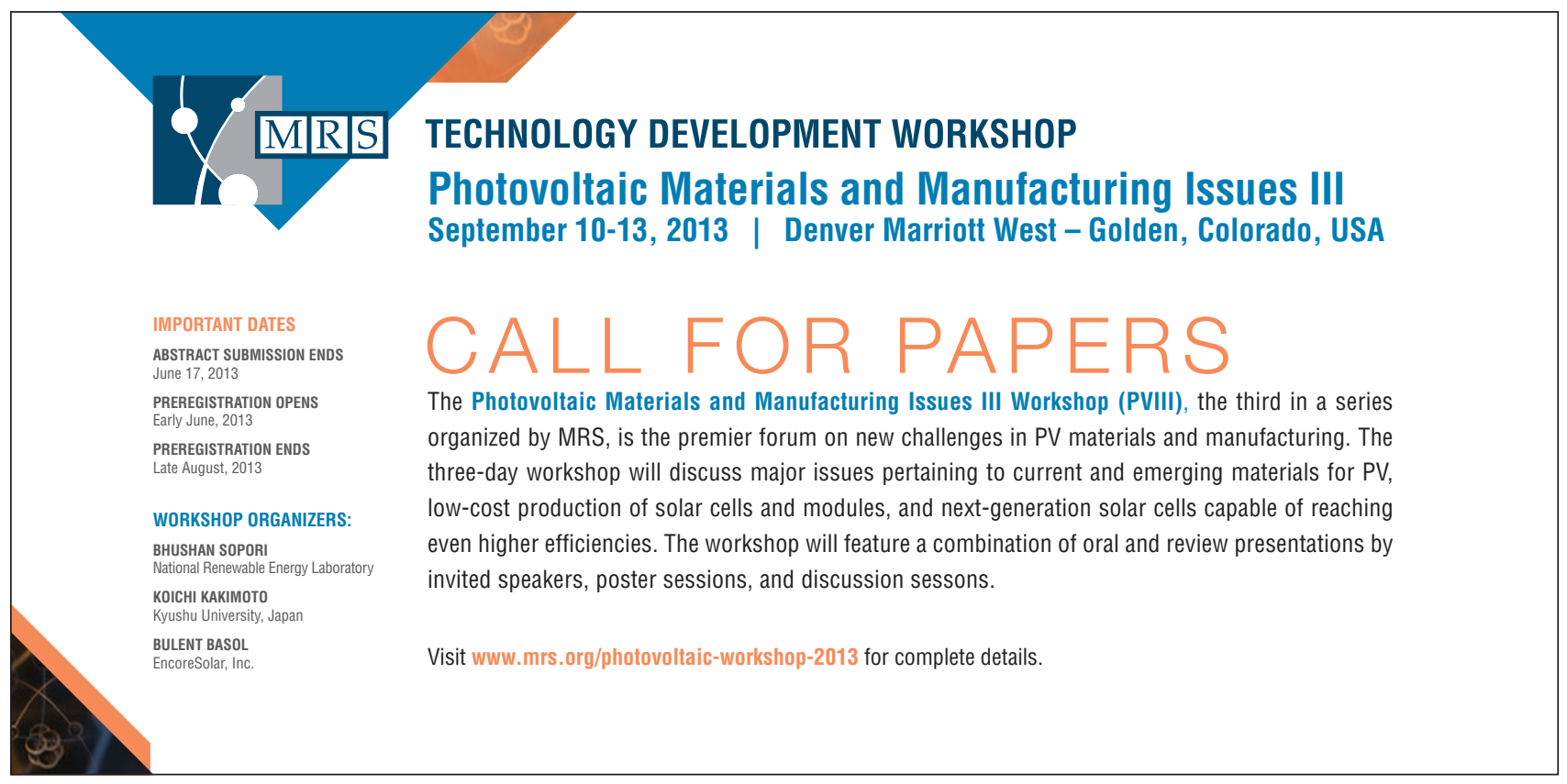

